\title{
Classification of N-Screen Services, Scenarios and its Standardization
}

\author{
Changwoo Yoon, Hyunwoo Lee, Won Ryu \\ *Electronics \& Telecommunications Research Institute, Daejeon, Korea \\ cwyoon@etri.re.kr, hwlee@etri.re.kr, wlyu@etri.re.kr
}

\begin{abstract}
By the advent of IPTV and smart TV, the broadcasting is transmitted using Internet. Bi-directional programs are appeared on broadcasting services. The convergence service combining with communication, information and web service are appeared too.

$\mathrm{N}$-Screen service is a killer service of smart TV. It uses several terminals, either fixed or mobile, to provide bi-directional, convergence and personal services with broadcasting service.

$\mathrm{N}$-Screen service can be classified into three categories: first, OSMU (One Source Multi Use) case, providing same contents to terminals having different capabilities such as screen size, CPU speed, memory, codec, network speed, etc. Second case is a vertical handover, continuous watching of content using different terminal. Third case is a collaborative service among multiple terminals. For example, a customer is watching soap opera using TV, while watching a specific scene related information or advertisement using his PAD or smart phone.

In ITU-T SG13, the Y.sof (Service Scenario over FMC) was standardized. It defined detailed overall service scenarios using feature extraction of seamless mobile convergence service on several networks such as WiFi, 3G, WiMAX/WiBro. This standard extracts key features of five key elements: person, terminal, network, content, and service. Then, it analyzes relationships among key elements and suggests overall service scenario model.

The service scenario model can be easily adopted on describing $\mathrm{N}$-Screen service scenario because $\mathrm{Y}$.sof handles scenario cases among several fixed or mobile terminals.

In this paper, I will introduce Y.sof and classification of N-Screen service scenarios described using the standard. Also I will refer the standardization issues of $\mathrm{N}$-Screen and its technologies.
\end{abstract}

Keywords - IPTV, Smart TV, N-Screen, OSMU, FMC

\section{INTRODUCTION}

Recently, the environment where consumers use multiple devices according to time and place is being created. In this situation, multi-device platforms are gaining attention for

Manuscript received September 15, 2012. This work was supported in part by the EU ITEA-2 project with Grant No.10028 "Web-of-Objects" (WoO) funded by MKE and supervised by KIAT.

Changwoo Yoon is Principle Researcher with the Electronics \& Telecommunications Research Institute, Daejeon, Korea (Phone: +82-42-8606543; fax: +82-42-860-5611; e-mail: cwyoon@etri.re.kr).

Hyunwoo Lee is Team Leader with the Electronics \& Telecommunications Research Institute, Daejeon, Korea (Phone: +82-42-860-6526; fax: +82-42861-1342; e-mail: hwlee@etri.re.kr).

Won Ryu is Director with the Electronics \& Telecommunications Research Institute, Daejeon, Korea (Phone: +82-42-860-6290; fax: +82-42861-1342; e-mail: wlyu@etri.re.kr). enabling users to enjoy the same content or services seamlessly, irrespective of which device / medium is used. N Screen which is recently getting attention falls into the broader concept of Multi-Device Service. N Screen is about enabling the user to use multiple devices, which means, it should be made up of integrated platforms for multi-devices. The core element of $\mathrm{N}$ Screen Service is a platform that mediates the use of content or services on multiple devices. [1,2]

N Screen Services and Multi-Device Services are often used in the same meaning. Technically speaking, however, Multi-Device Service is a broader concept that encompasses N Screen Service. Multi-Device Service is, literally, to provide the same content or services on a variety of devices. Along with the evolution of the ICT environment, Multi-Device Services have been evolving as well.

ITU-T Y.2720 Sup.14 describes overall scenario model for various services over FMC. In the overall scenario model, shown in Fig. 1, the red dotted box describes $\mathrm{N}$ - screen service scenario situation of an end user using his/her terminal devices while the features of service are operated on several devices synchronously. [3,4,5] An example is the case of displaying the same content on different types of terminals converting content quality. Another scenario is of an end user using his/her terminal devices while the unit feature of converged service is operated on separate devices synchronously. Examples are the case of displaying VOD on TV+STB, starting VOD using EPG displayed on mobile phone, and displaying VOD related information on a Notebook.

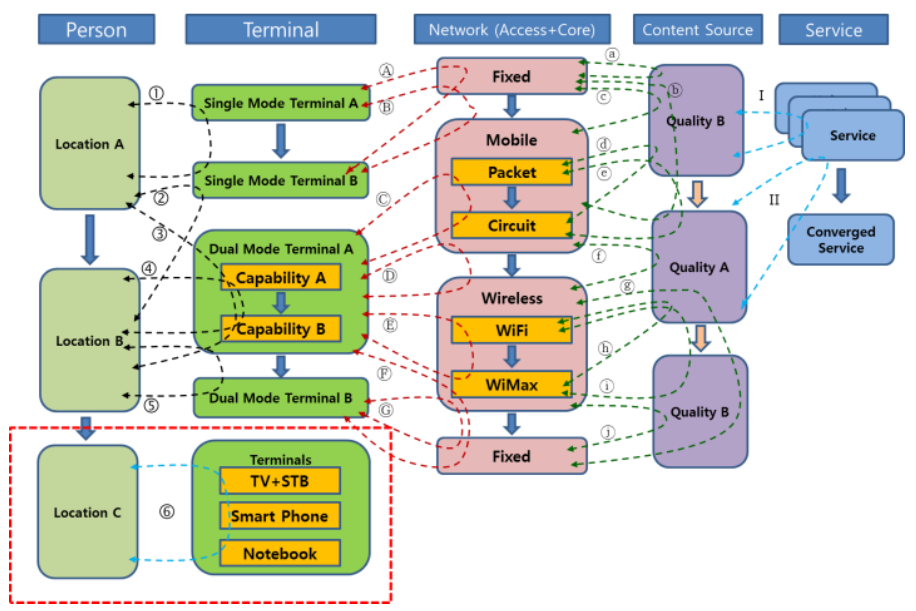

Figure 1. N-Screen part on Overall scenario model over FMC 
In this paper, we describe various kinds of $\mathrm{N}$-screen service scenarios based on overall scenario model over FMC.

\section{GENERAL DESCRIPTION OF N-SCREEN SERVICES}

By the advent of IPTV and smart TV, the broadcasting through Internet is generalized. The bi-directional service is introduced on broadcasting by using Internet's bi-directional transmission characteristic. The convergence services combined with telecommunication, information, web and personalized broadcasting services are appeared. Major feature of smart TV is intelligence such as smart search, extraction and UI technology providing customer targeted information on the limited size of TV screen. N-Screen service is a killer service of smart TV providing various kinds of bi-directional, converged, personalized and intelligent contents and services to multiple fixed or mobile devices

We can classify $\mathrm{N}$-screen service scenarios as three cases. First case is sharing same content or service on more than one screen among multiple kinds of screen; For example, it is a service on which an end user can watch the same content on various terminals such as TVs with Setop, PCs, notebooks, PMPs, or smart phones.

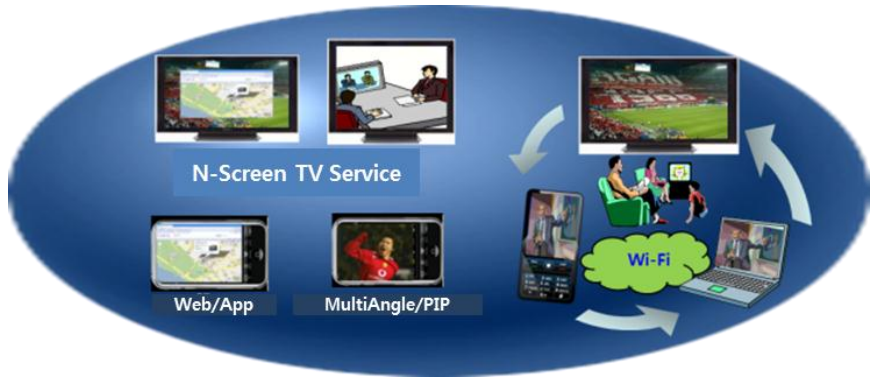

Figure 2. Classification Case I of N-Screen Service: OSMU

Another example of OSMU is migration of service among terminals. Figure 3 shows an example of OSMU of service. User can use smart TV application showing maps of specific location, while the user want to send an e-mail to her/his friend. Because it is difficult to write an e-mail on smart TV, the user migrate the e-mail window to his smartphone.

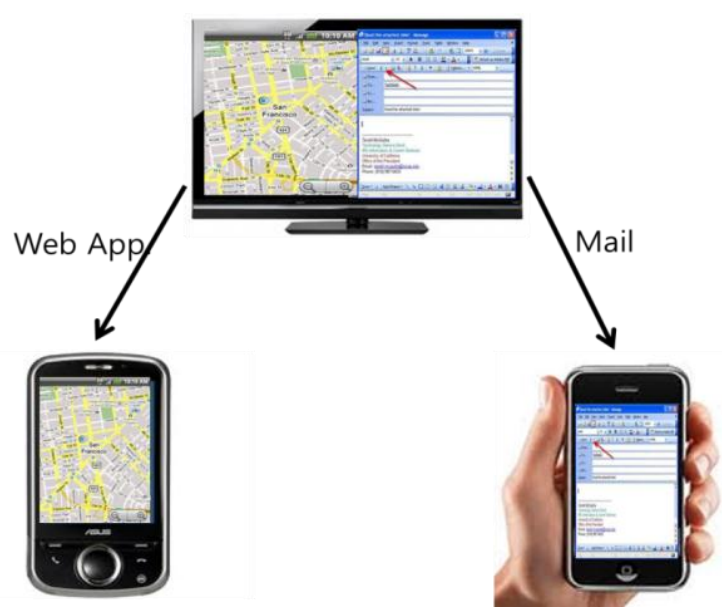

Figure 3. Classification Case I of N-Screen Service: OSMU of service
Second case is consuming same contents on several devices continuously. . It is considered as one of the representative service supporting service mobility among multiple kinds of screens.

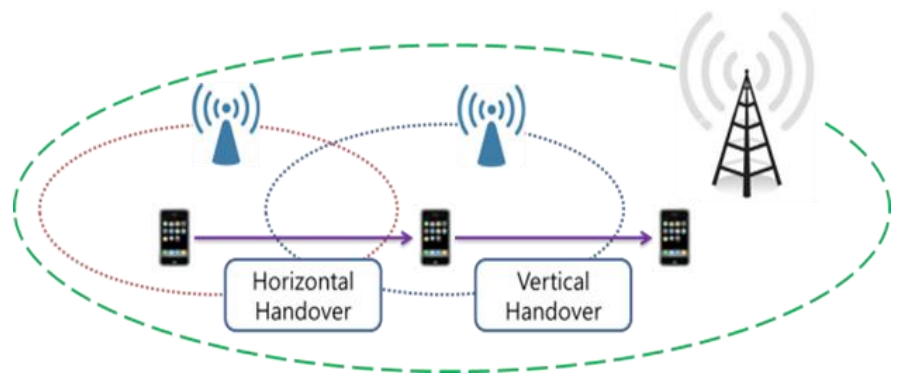

Figure 4. Classification Case II of N-Screen Service: Handover

Third case is providing collaborating service among multiple devices. The service provides to customer is consist of different shape of services that is operated on separate screen. For example, PAD operates as EPG terminal for an IPTV. A customer can select a program he wants to see and the selected program is displayed on TV screen with synchronous manner.

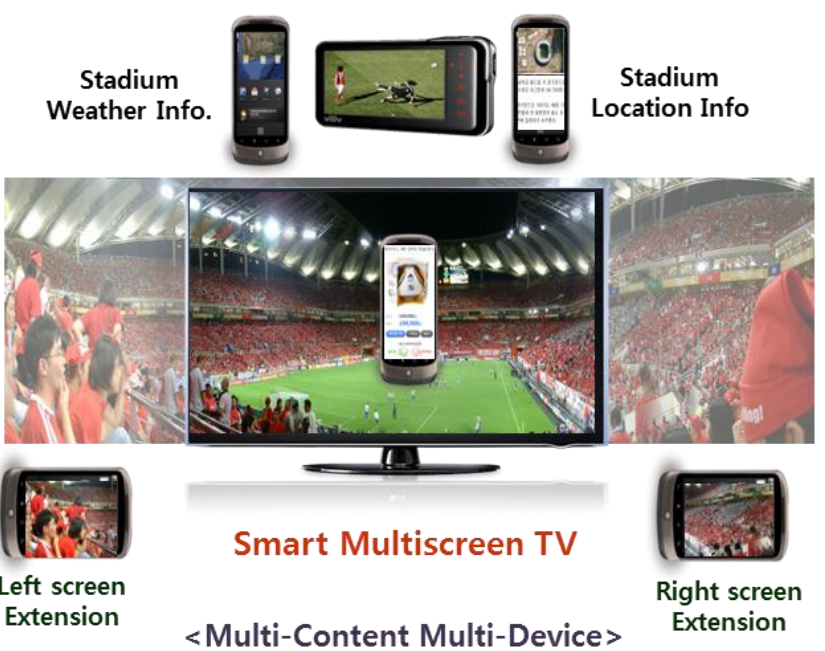

Figure 5. Classification Case I of N-Screen Service: Collaboration

More advanced example of case III collaboration is ASMD (Adaptive Source Multi-Device) shown on figure 6. In ASMD service scenario, user can divide and combine services making convergence services. In figure 6, three persons are watching TV while they are using their own handheld device such as mobile phone and pad. While watching music program, each person are using music program related services. Person A watches different angle scenes. Person B watches celebrity news about the singer who is showed in TV screen. Person C watches album about the singer. By a touch of each person's handheld device, the services shown in each person's device transmitted to big TV screen and displayed in combined mode.

This ASMD type of N-Screen service will bring new watching pattern on smart TV by providing collaborating TV services with mobile devices. There will bring new business 
opportunities by providing convergence services combining broadcasting, telecommunications and web service freely.

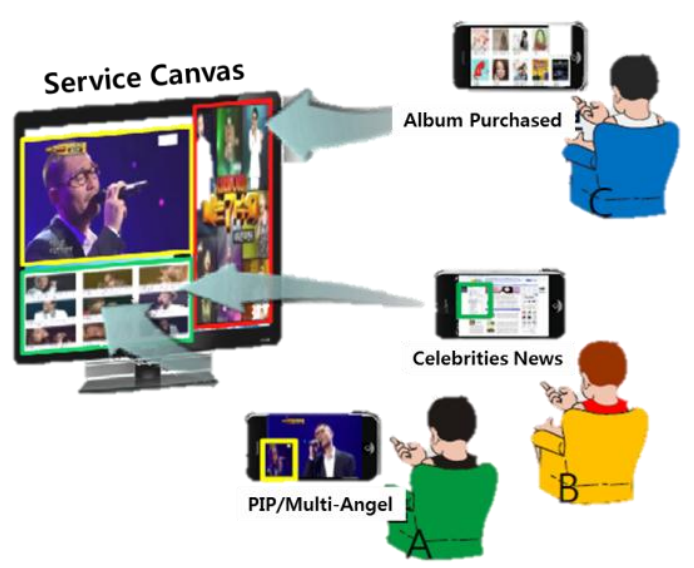

Figure 6. Classification Case III of N-Screen Service: ASMD

\section{Y.SOF: SERVICE SCENARIOS OVER FMC}

One of the essential benefits of the NGN is a supporting of convergences such as a fixed mobile convergence (called FMC), telecom-broadcasting converged service like IPTV. ITU-T produced several Recommendations on FMC and IPTV, especially in the ITU-T Y.2000 series of Recommendations. ITU-T is developing more detailed aspects of supporting the FMC, taking consideration of the fact that various services are waiting to utilize the FMC as their service infrastructure, which will extend their service coverage as well as give more benefits to the user.

This Supplement to the ITU-T Y.2000-series Recommendations on the scope of service scenarios over the FMC provides service scenarios which are used over FMC. This Supplement uses the features of involved key elements of FMC to guide how services can be provided in detail. This Supplement also introduces overall configurations and scenario models to identify service scenarios over the FMC.

There are five key elements to define: Person, Terminal, Network, Contents and Service.

- Person (End User): This is a key subject for consuming services over FMC. It is characterized by the location, that is, "Same Location" or "Change Location"

- Terminal: This is a key device supporting services for the person. The person can own multiple terminal devices either fixed, mobile or Wireless. The terminal can be classified into two categories (Single mode and Multi mode) by the number of network connection interfaces. As single mode terminal provides a single connection to the network that is either fixed or mobile or wireless. A multi-mode terminal provides several ways of connection to the networks. In this supplement, we use a dual mode terminal as an example of a multi mode terminal. The dual mode terminal provides dual connections to the network that are either fixed, mobile or wireless.
- Networks: This is a key part supporting mobility and FMC of the terminal. The network is composed of an access network and a core network. We can classify the access network by its technology basis, whether it is "Fixed", "Mobile" or "Wireless".

- Contents: This is a key part that is presented by media files and media processing. The contents form services for the end user.

- Service: This is a key part for the end users providing a set of functionalities enabled by a service provider.

I will describe the features of key element bellows.

\section{A. Behavioural aspect of Person}

A person will use terminal device either keeping the same location or changing location. Changing the location causes supporting of mobility if the person wishes to keep current services. So we can determine the features of the person's key element by the location, whether it is, "Same location" or "Change location".

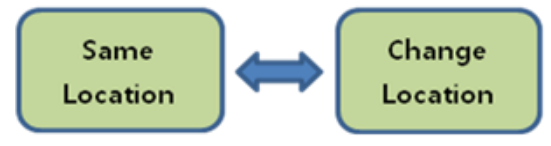

Figure 7. Features of Person

\section{B. Capability aspect of Terminal}

The terminal is operated following the behaviour of the person while trying to keep the service continuity. As a result of following person's behaviour, it is decided whether the capability of the terminal should be changed or not.

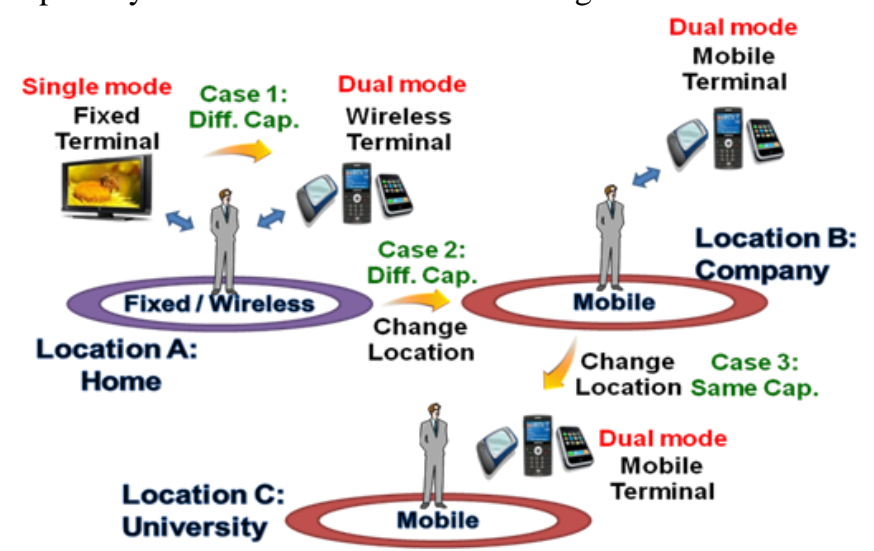

Figure 8. Example cases of Person's behaviour of terminal usage

For example, figure 8 shows three cases of the terminal capability change or no-change according to the behaviour of the person.

First case shows the situation of the person watching high quality IPTV at home. He has to go to his company and he wants to watch the program seamlessly using his mobile phone. In this case the capability of the terminal is changed: from single mode, fixed network TV to dual mode, wireless network mobile phone.

Second case shows the situation of the person moving his location from A (home, wireless network area) to B (company, 
mobile network area), while continuing the service. In this case the capability of the terminal is changed: network mode change of mobile phone from wireless network to mobile network.

Third case shows the situation of the person moving his location from B (company, mobile network area) to C (university, mobile network area), while continuing the service. In this case, there is no terminal capability change because it is merely a change of mobile base station.

Therefore we can determine the features of the terminal key element by the capability, whether it is, the "Same capability" or "Different capability".

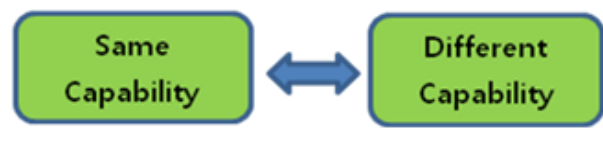

Figure 9. Features of Terminal

\section{Capability aspect of Network}

The features of access network needs to be considered by the technology basis: Fixed, Mobile and Wireless. For those networks, the network capability may vary from one network to another. For example, a fixed broadband network is able to support much higher bandwidth than cellular wireless.

Core network is a delivery part managing overall traffic transferring process such as re-routing, traffic congestion and failure in the routing path etc. The core network's capability should be impacted by end user behaviour such as changing access networks. Therefore, core network features can be determined by "Same Capability" and "Different Capability".

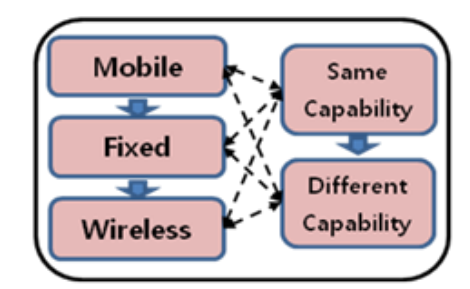

Figure 10.

Features of Network

\section{Quality aspect of Contents}

We can determine features of the content by its quality such as trans-coding QoS parameters, such as encoding codec, resolution (CIF, SD, HD) or frame rate

- Same quality: This is the case in which the source of contents should maintain the quality. From the contents point of view, there is no need to change trans-coding QoS parameters such as codec, resolution or frame rate.

- Different quality: This is the case in which the source of contents should change the quality. From the contents point of view, there is a need to change the trans-coding QoS parameters such as codec, resolution or frame rate.

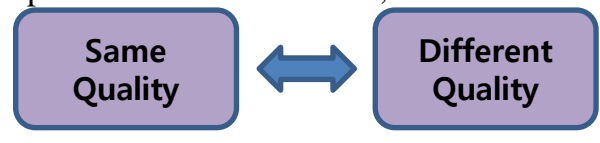

Figure 11.

Features of Contents
There are two cases for the Different quality: upgrade or downgrade.

Example case for the upgrade is a handover from a mobile terminal to a TV requiring high quality video and network. The change of quality is required such as codec and resolution (CIF to HD).

Example case for the downgrade is a handover from a TV to a mobile terminal. The change of quality is required such as codec and resolution (HD to CIF).

\section{E. Integration aspect of Service}

Service feature can be classified by its integration, whether it is, "service" or "Converged service". A converged service can be composed of several services.

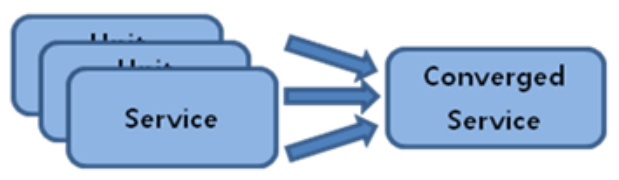

Figure 12. Features of Service

\section{F. Overall FMC configuration model}

This clause shows an overall high level configuration model over FMC. This is determined by considering the features of FMC key elements, and their characteristics.

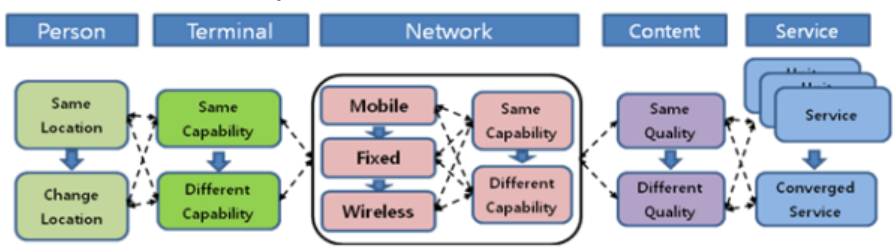

Figure 13. Overall configuration model using features of key elements

In this figure, a person will use his/her terminal device either maintaining the same location or changing the location. Changing the location demands support of mobility if the person wishes to keep services while moving.

Then, a terminal device (either single mode or multiple modes) is operated to follow the behaviour of the person while keeping the network connection as much as possible. As a result of following user behaviour (handover to other terminal or change of network connection), the capability of the terminal function should be changed.

In the case of access networks, it should be continuously changed according to the end user's behaviour such as moving or changing the connection among fixed, mobile and wireless access networks. One important thing here is that changing the access network causes change in the connecting capability like bandwidth, or overall traffic management process.

Sources of contents are influenced by the mobility, because the result of mobility either caused at terminal device or access network requires changing the QoS (downgrading or upgrading). Therefore this can be characterized by the quality: "Same Quality" or "Different Quality."

Service providers provide several types of service such as content on demand, real-time broadcasting IPTV service, IMS based caller ID service, information display etc. Service 
providers can provide converged services by combining services such as displaying caller-ID, content related information while the customer is watching VOD.

Using overall FMC configuration model, we can derive overall scenario model over FMC shown in Figure 1.

In figure 1, scenario number 6 shows $\mathrm{N}$-screen scenario case. This is a scenario of an end user using his/her terminal devices while the features of service are operated on several devices synchronously. An example is the case of displaying the same content on different types of terminals converting content quality. Another scenario is of an end user using his/her terminal devices while the unit feature of converged service is operated on separate devices synchronously. Examples are the case of displaying VOD on TV+STB, starting VOD using EPG displayed on mobile phone, and displaying VOD related information on a Notebook.

\section{IV.N-SCREEN SERVICE SCENARIO: CASE I}

Case I N-screen is a service on which an end user can watch the same content on various terminals such as TVs with Setop, PCs, notebooks, PMPs, or smart phones. Each screen of terminals is used in a cooperative and synchronous manner.

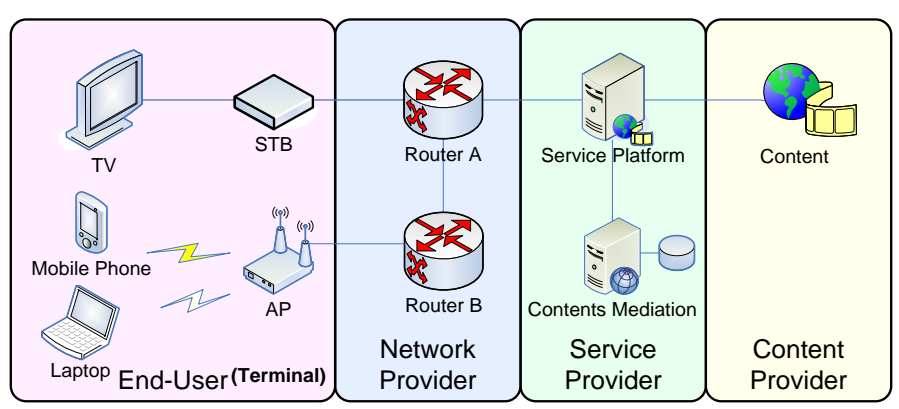

Figure 14. Overall configuration of N-screen case watching same content

Figure 14 is a 3 -screen service with content sharing. Three screen service allows an IPTV service subscriber consume IPTV service contents on TV, PC, and wireless screens. The basic type of 3 -screen service is sharing the same IPTV service contents on more than one screen among the three kinds of screens.

\section{A. Service scenarios of $N$-screen service case watching same content on multi-devices}

1. [Service Provider $>$ End-user(Person): watching Nscreen service on Terminal A] End-user(Person) is watching contents provided by content delivery functions of a service provider via network functions of a network provider using terminal A (For example, a TV with setop). The end-user may have a plan to watch the content using terminal B (For example, a Laptop) and Terminal C (For example, a mobile phone) at the same time.

2. [End-user(Person)-> Service Provider: request Nscreen service to Terminal B,C] End user functions of the end-user request the terminal to provide current service to terminal B.

3. [Service Provider: preparing N-screen service for terminal B \& C] The N-screen service requested to display same content of terminal A to terminal B, C is processed by service provider's service control function. The service control functions of the service provider adjust quality of contents to network bandwidth to be changed. The service control functions decide to transcode origin contents into suitable contents to be changed, considering display sizes to be changed. Application functions of the service provider may have contents mediation functions like codec convertors. The application functions converts content into small-size or big-size display, depending on the device profile and access network bandwidths.

4. [Service Provider -> Terminal B,C -> End user(Person): using N-screen service] The newly generated contents are delivered into designated storage managed by content delivery functions. The content delivery functions send adjusted contents to terminal B and C.

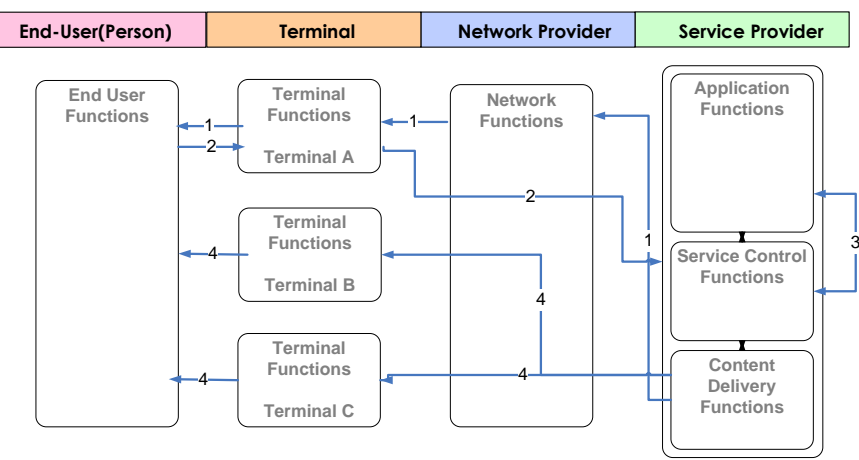

Figure 15. Service scenario of N-screen case watching same content

\section{N-SCREEN SERVICE SCENARIO: CASE II}

Case II $\mathrm{N}$-screen is consuming same contents on several devices continuously. It is considered as one of the representative service supporting service mobility among multiple kinds of screens. Figure 16 is one of the examples of case II N-screen service. Below is the service scenario of case II N-screen: service continuity.

\section{A. Service scenarios of $\mathrm{N}$-screen service case consuming same content on multi-devices continuously.}

1. [Service Provider-> End-user(Person): watching Nscreen service on Terminal A] End-user (Person) is watching contents provided by content delivery functions of a service provider via network functions of a network provider using terminal A (For example, a TV with STB in a living room). 


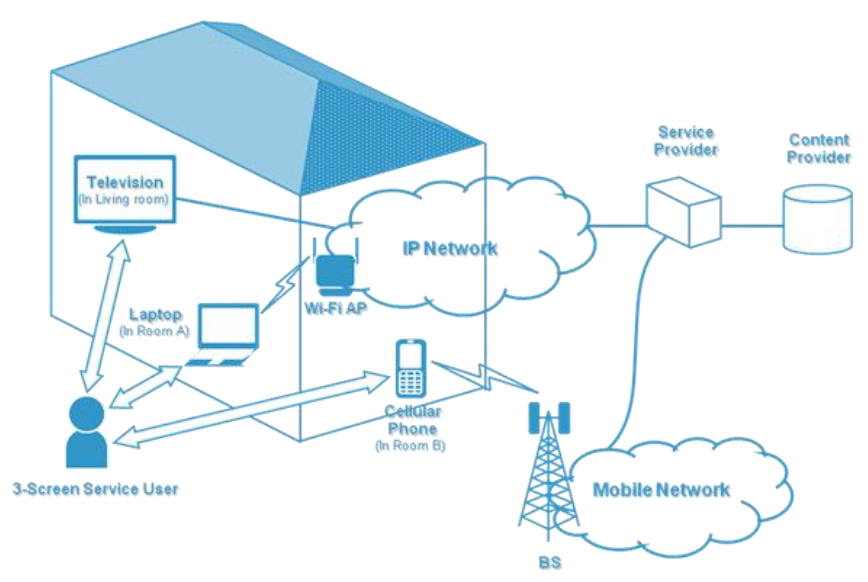

Figure 16. 3-screen service with service continuity

2. [End-user(Person): moving N-screen service on Terminal B] The end-user moves from living room to room A to watch the same content continuously using terminal B (For example, a Laptop).

3. [Terminal B-> Service Provider: request $\mathrm{N}$-screen service to Terminal B] Terminal B is authenticated as an $\mathrm{N}$-screen service and requests the service provider to send the same content.

4. [Service Provider: preparing N-screen service for terminal B] The $\mathrm{N}$-screen service requested to display same content continuously on the terminal B is processed by service provider's service control function. The service control functions of the service provider adjust quality of contents to network bandwidth to be changed. The service control functions decide to transcode origin contents into suitable contents to be changed, considering display sizes to be changed. Application functions of the service provider may have contents mediation functions like codec convertors. The application functions converts content into small-size or big-size display, depending on the device profile and access network bandwidths.

5. [Service Provider -> Terminal B -> End user(Person): using $\mathrm{N}$-screen service] The newly generated contents are delivered into designated storage managed by content delivery functions. The content delivery functions send adjusted contents to terminal B.

6. [End-user(Person): moving N-screen service on Terminal C] The end-user moves from room A to room B to watch the same content continuously using terminal C (For example, a Cellular phone). The terminal C shows the same content continuously through the same process as described above.

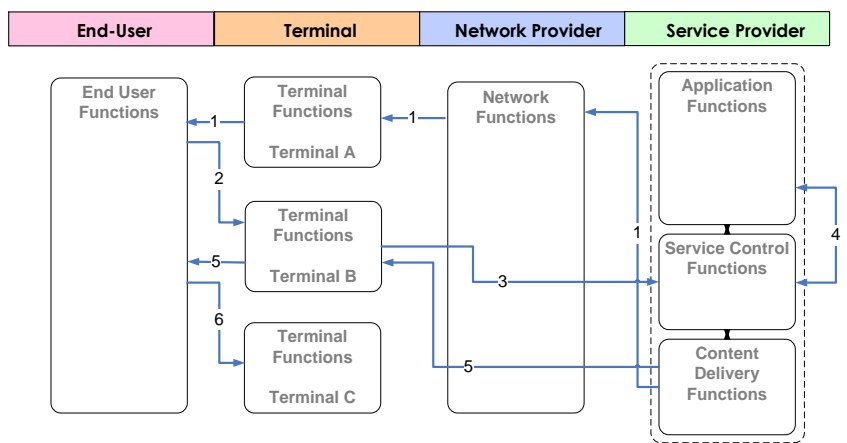

Figure 17. Service scenario of N-screen case consuming same content on multi-devices continuously

\section{VI.SCENARIO FOR N-SCREEN SERVICE CASE III SCENARIO 1: TARGETED ADVERTISING SERVICE TO SEPARATE TERMINAL}

I will describe $\mathrm{N}$-screen service scenario case III example, targeted mobile advertisement service that is a collaborative case.

Targeted advertisement service is providing Ad to person's mobile terminal while he is watching VOD on TV screen. During watching a VOD channel, logging and selecting keyword may be required to initiate the service. Choosing 'keyword' plus 'interests' among menus may load Ad service web pages, which gathers related Ad contents and metadata from Web and 3rd party Ad server. The login user's interests are dependent on user profile. In case that the keyword belongs to 'people', the person may appear in aggregated commercial advertisement. As supplementary Ads, banner advertisement, which is inserted by advertiser's request, is located near targeted advertising service or other convergence services.

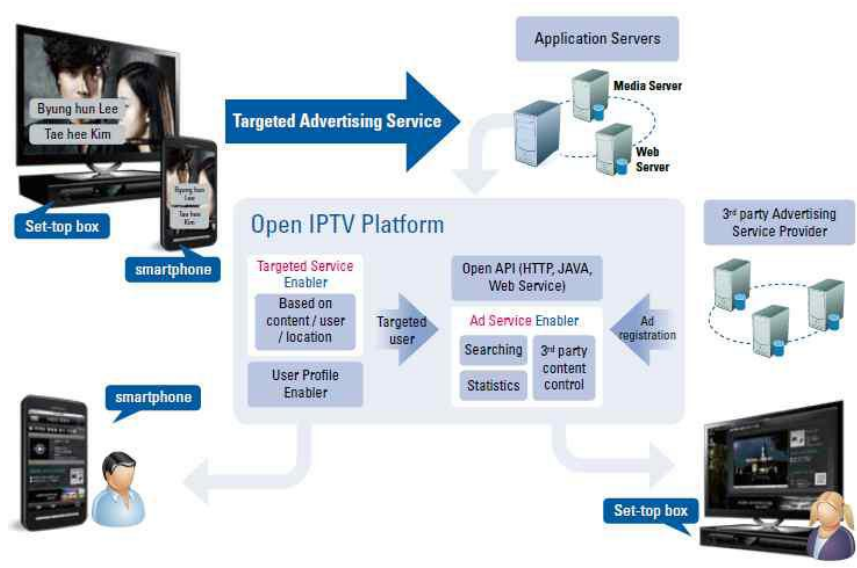

Figure 18. Targeted Advertising Service

The split EPG terminal has function to control IPTV service via home AP. A mobile user in the right of figure 18 watches golf sports channel in VOD service. He already gave his preference to the profile enabler via open service platform. For instance, since the profile enabler knows he enjoys golf as outdoor sports, when he detects golf driver on watching golf contents, the mobile ad process enabler may give him ad moving picture about newly released golf driver. Mobile ad 
process enabler may give helpful information to him as well as connect to the purchase step by the mobile device.

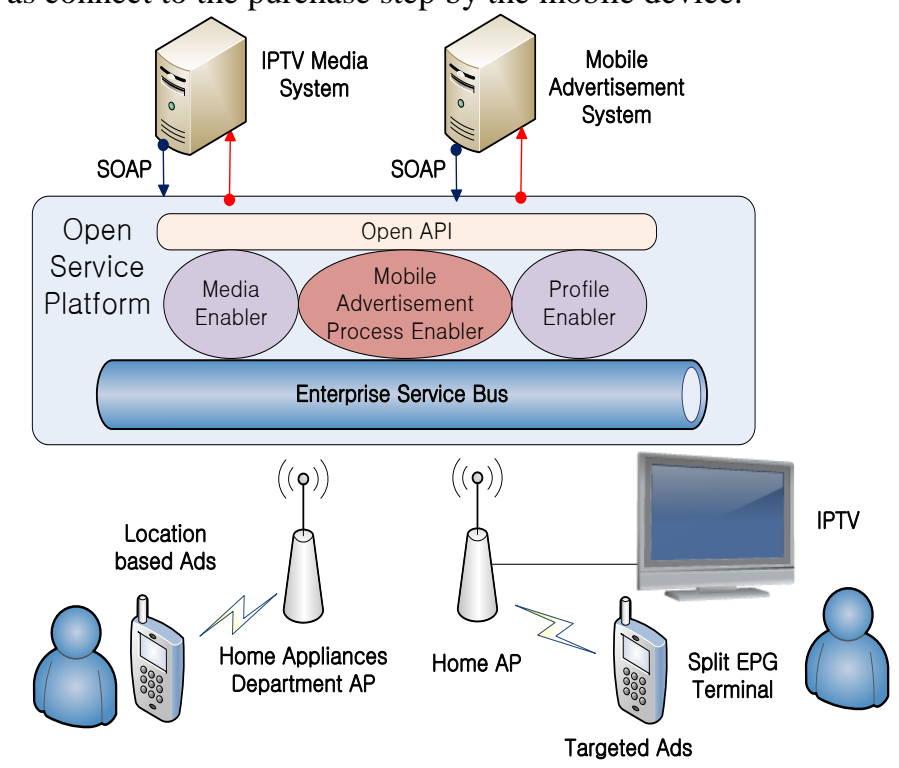

Figure 19. Example system of Targeted Advertising Service

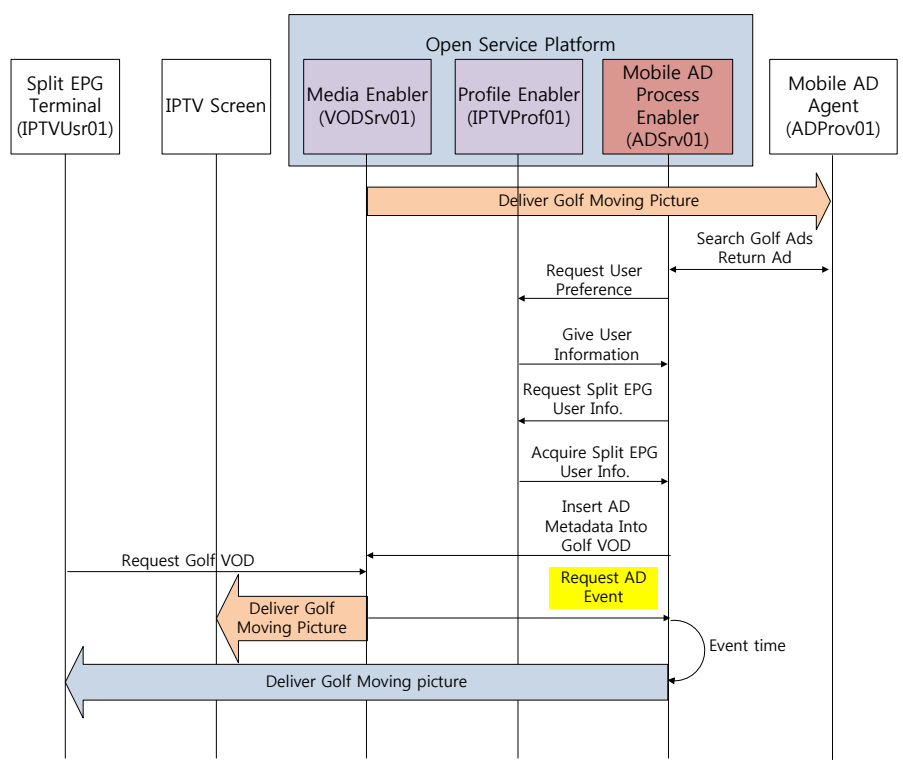

Figure 20. Service flows of Targeted Advertising Service

Figure 19 shows example of targeted advertisement using SDP. Mobile ad agent may be person or sub-system of an ad service provider. He also watches a golf VOD service via media enabler within open service platform. The mobile ad process enabler searches a golf ad among many ad contents of a mobile ad agent. That golf ad is related with revealed product in golf moving picture. The mobile ad process enabler acquires user preference and whether mobile users own split EPG terminals. The convergence enabler then inserts ad metadata into golf VOD. A mobile user with split EPG terminal requests to view that golf contents to open service platform. As soon as golf moving picture is delivered to an IPTV user, ad request event is generated.

\section{A. Service scenarios of $\mathbf{N}$-screen service case collaborating convergence service: targeted advertising service to separate terminal.}

1. [Service Provider -> End-user(Person): watching VOD channel on Terminal A] End-User(Person) is watching VOD channel with Ad service which is provided content delivery functions of service provider via network functions of network provider using terminal A (For example, a TV with setop). We suppose that end-user with Terminal A is a woman in forties and she likes to collect jewelry.

2. [End-user(Person) -> Service Provider: initiate Targeted advertisement service on Terminal A] The end-user can login and select keyword to initiate the targeted advertisement service. By choosing 'keyword' plus 'interests' on menues of Terminal A, related Ad contents and metadata from Web and 3RD party Ad Server is gathered by service control functions of the service provider.

3. [Service Provider: prefaring and generate targeted web pages for Terminal A] Targeted advertised content is generated with gathering Ad contents , metadata and profile information of end-user with Terminal A

4. [Service Provider -> Terminal A-> End user(Person): using targeted advertised service with Terminal A] The newly generated content which has targeted information(For example, jewelry shops) for end-user with Terminal A is delivered to Terminal A by content delivery functions.

5. [End user(Person) -> Service Provider : initiate Targeted advertisement service on Terminal B] EndUser(Person) has a hand-helded device such as smartphone. He lives with his mother who is enduser using Terminal A. Let's suppose that end-user with Terminal B is a man in twenties and he is interested in car so much. He can also initiate targeted advertised service by logining and selecting keyword. At this time, user profile information of end-user with Terminal B is transmitted to the service control functions of the service provider. Related Ad contents and metadata for end-user with Terminal B from Web and 3RD party Ad Server is also gathered by service control functions of the service provider.

6. [Service Provider: prefaring and generate targeted web pages for Terminal B] Targeted advertised content is generated with gathering Ad contents , metadata and profile information of end-user with Terminal B 
7. [Service Provider -> Terminal A-> End user(Person): using targeted advertised service with Terminal B] The newly generated content which has targeted information(For example, car Ad) for enduser with Terminal B is delivered to Terminal B by content delivery functions.

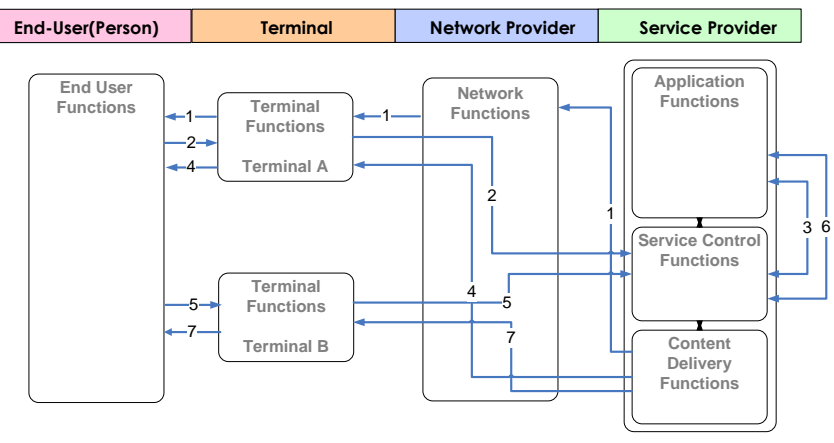

Figure 21. Service scenario of N-screen case using targeted advertising service

\section{N-SCREEN SERVICE SCENARIO CASE III SCENARIO 3: ASMD}

ASMD (Adaptive Source Multi-Device) is a collaborative case of N-screen service. The concept of collaborative case of $\mathrm{N}$-screen is an existence of collaboration among multi-screen showing different content or services for screens. The ASMD adds content/service adaption concept upon the collaboration. For example, if a user watching TV content related news using his mobile phone, then he wants to transfer the news to big TV screen to share the news with his family. Because the size and resolution is different between TV and mobile phone, proper adaption should be done on service presentation. In ASMD type of collaborative $\mathrm{N}$-screen service, it is not sufficient to provide service only by collaboration.

\section{A. Service scenarios of $\mathrm{N}$-screen service case III collaborating: ASMD}

1. [Service Provider $>$ End-user(Person): watching TV program(baseball) on Terminal A(Big-screen TV)] User1, 2, 3 (3 Persons) are watching TV program on Terminal A(Big-screen TV) provided by content delivery functions of a service provider via network functions of a network provider.

2. [Service Provider $>$ End-user(Person): using service on Terminal] User1, 2, 3 (3 Persons) are using services on their own handheld device (Terminal B, C, D). The services are related with TV program shown on Terminal A (Big-screen TV). For example, User 1 watches multi-angle scene of the baseball game on his smartphone. User 2 watches shorts news about the played baseball game on his tablet. User 3 uses baseball-game statistics service.

3. [End-user(Person)-> Service Provider: request sending their services to Terminal A] End user functions of the end-user requests their own terminal $\mathrm{B}, \mathrm{C}, \mathrm{D}$ to transfer current service to terminal A to discuss about the game by watching each person's service together. The $\mathrm{N}$-screen service requested is to display combined contents of services of terminal B, C, and D. The N-screen Service control function receives the request.

4. [Service Provider: preparing N-screen service for terminal A] The N-screen service control functions of the service provider adjust quality of services to fit the capabilities of different terminals. The Application function receives adapted content from service control function then sends it to terminal A using collaboration function of N-screen service control function.

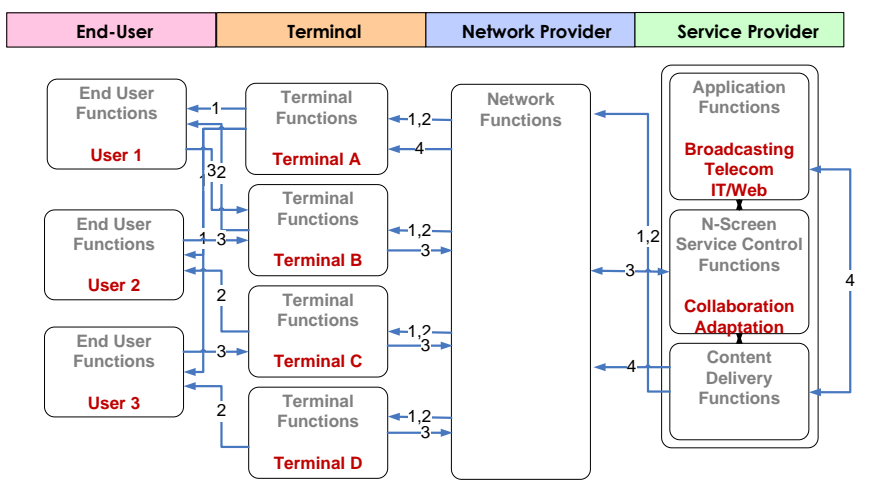

Figure 22. Service scenario of ASMD, N-screen collaborative case

\section{CONCLUSIONS}

The major keywords for N-screen service are cloud computing and social TV. The cloud computing is expending its technological importance and business area. It is possible to provide information synchronization service inside home and dynamic resource allocation using cloud computing's technologies such as virtualization, remote storage and mobile cloud [7]. Those kinds of services are closely related to Nscreen service.

It is important to combine with social TV to invigorate Nscreen service business. Using social TV concept, it is easy to introduce $\mathrm{N}$-screen into IPTV or smart TV because the customer can use collaborative services with main VOD or channel content. We described the targeted advertisement service that the customer watches advertisement content on different screen while he watches TV. At that scenario, we used service delivery platform to deliver IPTV and advertisement [8, 9, 10]

$\mathrm{N}$-screen service standardization is progressing through ITU-T Q24/SG13 for the service scenarios and use cases. The standardization of architecture and cloud computing related will be progressed.

In this paper, we described N-screen service concept, $\mathrm{N}$ screen service classification, service scenario description method, and service scenarios. Among three cases of N-screen service classification, the case III, collaborative case among 
several terminals is most importance in the aspect of business model. Right now, the telecommunication venders are focusing to provide OSMU and seamless case of N-screen. However the case III will catch user's attention because of its variety of service cases and features.

\section{REFERENCES}

[1] Changwoo Yoon, Hyunwoo Lee, Won Ryu, Bongtae Kim, "IPTV Service and Technology Evolutions," Journal of Korea Information and Communication Society, pp. 1-9, August, 2008.

[2] Changwoo Yoon, Shinmo Kim, Hyunwoo Lee, "Convergence Service Implementation based on Service Delivery Platform and Research Issues," International Technical Conference on Circuits/Systems, Computers and Communications, pp. 1080-1083, July 2009.

[3] ITU-T, Next Generation Network Global Standards initiative (NGNGSI), http://www.itu.int/ITU-T/ngn/index.phtml

[4] "Service scenarios over FMC," ITU-T Recommendation Series Y.2720 Supplement 14, 2011.1.

[5] "Supplement on IPTV service use cases," ITU-T Recommendation Series Y Supplement 5, 2008.

[6] "Web-based IPTV brokering service models and scenarios," ITU-T Draft Recommendation Series Y.iptvbs, September 2010.

[7] Changwoo Yoon, Mohammad Mehedi Hassan, Biao Song, Hyunwoo Lee, Won Ryu and Eui-Nam Huh, "Dynamic Collaborative Cloud Service Platform: Opportunities and Challenges," ETRI Journal, Vol.32, No.4, pp. 634-637, August 2010

[8] Changwoo Yoon, Hyunwoo Lee, Won Ryu, "Design of Layered service delivery platform for enabling I-centric convergence service," Communications and Information Technology, 2009. ISCIT 2009. 9th International Symposium on, pp. 243-247, September, 2009.

[9] Changwoo Yoon, Hyunwoo Lee, "Service delivery platform for convergence service creation and management," Proceeding ICACT'10 Proceedings of the $12^{\text {th }}$ international conference on Advanced communication technology, pp.1335-1338, Feb, 2010.

[10] Changwoo Yoon, Hyunwoo Lee, Won Ryu, "Next generation IPTV Platform," $20109^{\text {th }}$ International Conference on Optical Internet (COIN), pp. 1-3, July 2010.

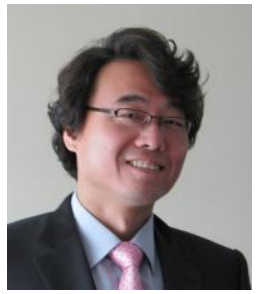

Changwoo Yoon received the B.S. degree from Sogang University, Seoul, Korea, in 1990. He received M.S. degree from POSTECH, Pohang, Korea, in 1992. He received Ph.D. degree in Computer \& Information Science \& Engineering from University of Florida, US, in 2005. Currently he is principal researcher in Creative Future Research team, ETRI and adjunct professor at UST. His current research interests include N-Screen, IPTV, Cloud computing, SOA, Service creation/delivery technology and information retrieval.

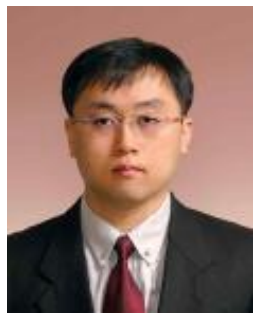

Hyunwoo Lee received M.S. and Ph.D. degrees in 1995 and 2005, respectively, in Korea Aerospace University (KAU). He is currently a principal research engineer and team leader in convergence service networking research team, smart screen convergence research department, ETRI. His main research interests include heterogeneous wireless access network, Mobile P2P, open IPTV platform in NGN. His current research interests include cloud computing and platform.

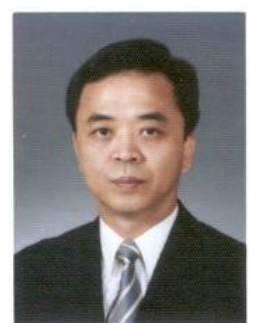

Won Ryu, Ph.D received the BS degree in computer science and statistics from Pusan National University, Busan, South Korea, in 1983, and the MS degree in computer science and statistics from Seoul National University, Seoul, South Korea, in 1987. He received his $\mathrm{PhD}$ degree in information engineering from Sungkyunkwan University, Kyonggi, South Korea, in 2000. Since 1989, he has been a managing director with the Smart screen convergence research department, ETRI, Daejeon, Korea. Currently, his research interests are IPTV, Smart TV, IMT-advanced, and convergence services and networks and etc. 